\title{
FÜNFZIG JAHRE SCHWEIZERISCHES ALPINES MUSEUM
}

\section{PAUL Köchli}

Vor fünfzig Jahren konnte in Bern das erste schweizerische alpine Museum eröffnet werden. Anläßlich des diesjährigen Jubiläums ist es wohl gestattet, einen Blick auf Entstehung, Wirken und Ziele des Museums zu werfen.

Der Gedanke, ein solches Museum zu gründen, läßt sich weitgehend aus der damaligen Anteilnahme an den bergsteigerischen Leistungen und wissenschaftlichen $\mathrm{Er}$ kenntnissen und Theorien, besonders auch über die Entstehung der Hochgebirge, erklären. Eine Wirkung davon war, daß sich der Schweizer Alpenclub an der Landesausstellung 1896 in Genf und an der bernischen kantonalen Gewerbe-, Industrie- und landwirtschaftlichen Ausstellung 1899 in Thun mit einer Sonderschau über die Gebirgswelt beteiligte. Das für diese Ausstellungen mühsam gesammelte Material wurde leider nachher meistens wieder in alle Winde zerstreut und ging sogar teilweise für immer verloren. Diese betrüblichen Erfahrungen veranlaßten einige Berner Alpinisten, allen voran Paul Utinger und Dr. Rudolf Zeller, der Sektion Bern vorzuschlagen, ein alpines Museum zu schaffen. Am 9. Juli 1905 wurde nach der Überwindung zahlreicher Schwierigkeiten im alten Standesrathaus in Bern in wenigen Räumen das Museum eröffnet. Die Gestaltung stand unter der sachkundigen Leitung von Dr. ZELLER, dem späteren Ordinarius für Geographie an der Universität Bern. Schon bald wurde es von fachlicher Seite anerkannt. Im Geographischen Anzeiger 1908 schrieb Prof. M. Friederichsen u. a., es bleibe nach Abzug der sportlich-touristischen Abteilung ein «von echt geographischem Geiste getragenes länderkundliches Museum des Schweizer Alpengebietes»zurück. Und er fuhr fort: «Hier kommt es der $\mathrm{Mu}$ seumsleitung darauf an, wissenschaftlich fundierte Aufklärung über die Gesamtheit aller Erscheinungen der Natur der Schweizer Alpen in den Kreisen der Bergsteiger wie des großen Publikums zu verbreiten, Liebe und Verständnis für die Bergwelt zu wecken und zu vertiefen ».

Diesen Äußerungen Prof. Friederichsens fühlte sich die Museumsleitung stets verpflichtet. Es gelang ihr nach der Errichtung des Neubaues auf dem Kirchenfelde im Jahre 1934, den geographischen Anforderungen in größerem und besserem Ausmaße zu entsprechen. Der «Alpinismus» wird im Museum nicht als Bergsteigen im engeren Sinne ausgelegt, sondern umfaßt alles, was mit den Alpen in Beziehung steht. Gemäß dieser Ansicht gliedert sich das Museum in folgende Abteilungen:

1. Stock

\begin{tabular}{|c|c|c|c|}
\hline $\begin{array}{l}\text { Hochgebirgs- } \\
\text { forschung }\end{array}$ & \multicolumn{2}{|c|}{$\begin{array}{l}\text { Tier- und } \\
\text { Pflanzenwelt }\end{array}$} & $\begin{array}{l}\text { Schmetterlings- } \\
\text { sammlung }\end{array}$ \\
\hline \multicolumn{2}{|c|}{$\begin{array}{l}\text { Mineralogie und } \\
\text { Geologie }\end{array}$} & & $\begin{array}{l}\text { Persönliche } \\
\text { Ausrüstung }\end{array}$ \\
\hline $\begin{array}{l}\text { Gletscher- } \\
\text { kunde }\end{array}$ & & \multicolumn{2}{|c|}{$\begin{array}{l}\text { Geschichte des SAC } \\
\text { Hüttenwesen }\end{array}$} \\
\hline \multicolumn{4}{|c|}{$\begin{array}{c}\text { Führerwesen im Zusammenhang mit der } \\
\text { Erschließung der Alpen und } \\
\text { Rettungswesen }\end{array}$} \\
\hline
\end{tabular}

2. Stock

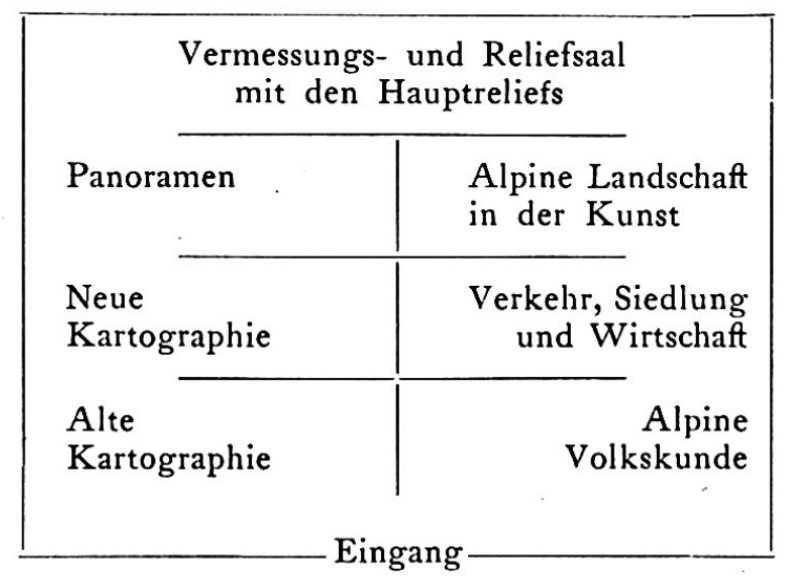

Anmerkung: Im Untergeschoß und Erdgeschoß befindet sich das Postmuseum.

Die einzelnen Abteilungen bilden in sich ein Ganzes und sind so aufgebaut, da $\beta$ auch der Laie die Zusammenhänge zu erfassen vermag. Soweit es die Platzverhältnisse 


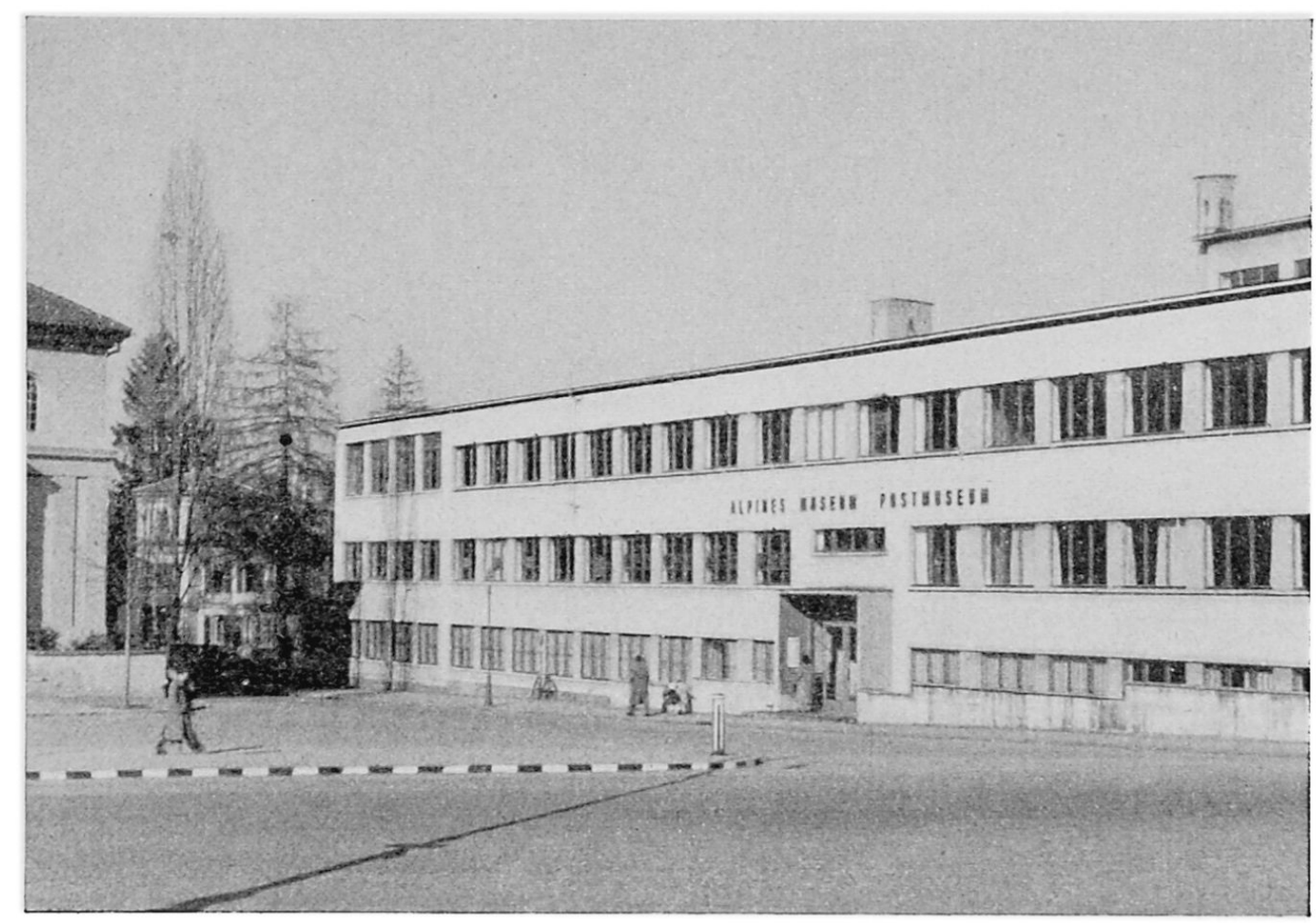

Abb. 1 Der Neubau des Schweizerischen Alpinen Museums von 1934/35 in Bern, Helvetiap'atz. (Aus W. Rıтz: Fünfzig Jahre Schweiz. Alpines Museum, Bern 1955.)

gestatten - jede Überladenheit soll möglichst vermieden werden - sind kurze Texte in deutscher und französischer Sprache zur Erklärung angebracht. Überall wird versucht darzustellen, wie sich aus dem Vergangenen das Heutige entwickelt hat, und wie sich Kommendes ankündigt.

Der gegenwärtige, verdiente Direktor des Museums, Prof. Dr. W. Rytz, vertritt die gleiche Auffassung wie seine Vorgänger, daß nämlich ein Museum, das nicht von Zeit zu Zeit neu organisiert werde, tot sei. So ist in den letzten Jahren die Abteilung für Rettungswesen neu gestaltet worden und zeigt heute die modernsten Rettungsgeräte in Wirklichkeit oder in Modellen. Ihr Einsatz wird anhand von Photographien erläutert. Die nächste große Aufgabe besteht darin, die hochalpine Forschung in allgemein verständlicher Form darzustellen; denn das Museum soll lebendige Wirklichkeit enthalten, um die Besucher anzuregen.

Von den Schätzen des Museums seien hier nur einige erwähnt, so vor allem die drei großen Bilder von CALAME (Staubbach bei Lauterbrunnen, Bergsturz im Haslital und Handeck), Winterlins «Blick ins Oberhaslital», DE Meurons «Lac de Brienz», Annelers «Lötschental» (ein Geschenk zum 50 jährigen Jubiläum) ; dann die Reliefs wie das von Simon über das Berner Oberland, von IMfeld das Matterhorn, von Alb. Heim den Pilatus, von Leo Aegerter den Dachstein und von IMHOF die Große Windgälle und das Bietschhorn. Der Gemeinderat von Grindelwald hat die Führerbücher seiner bekanntesten Bergführer, nämlich des Christian $A_{L}-$ MER, Vater, (1826-98) und seiner fünf Söhne (Christian, Ulrich, Hans, RuDOLF und PETER) hinterlegt, die alle ausgestellt sind und einzigartige Dokumente über Bergbesteigungen aus den damaligen Zeiten darstellen. Auch die Führerbücher des bekannten Aloys Burgener von Eisten/Saas und des Benjamin Ruvinez und des Joseph Furrer aus Sitten sind vorhanden. Wer sich besonders eingehend den Alpenhäusern widmen will, findet eine prachtvolle Photosammlung der Alpenhaustypen nebst einer Reihe von Modellen, so vor allem des bekannten Knuttihauses aus Därstetten und der Walliserhäuser aus dem Goms. 


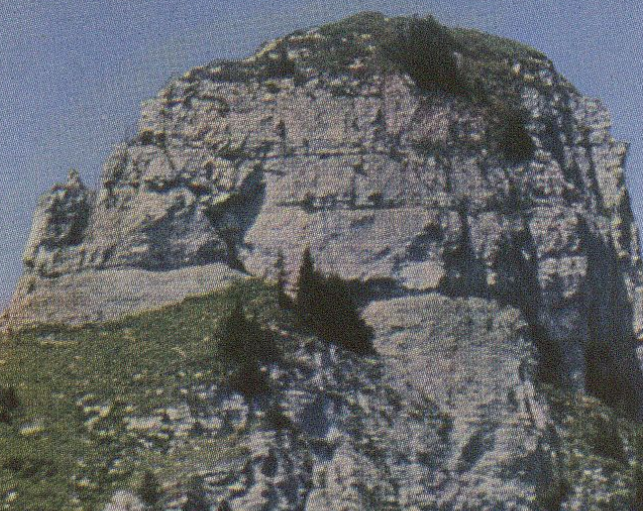

औ.

6.7.

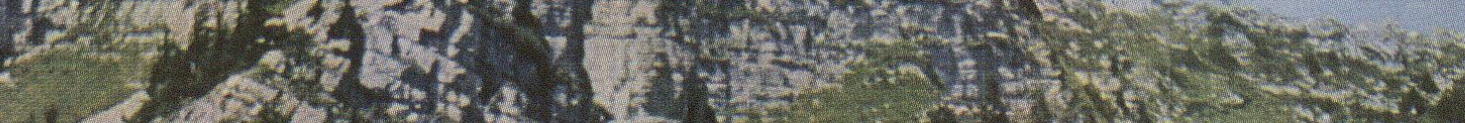

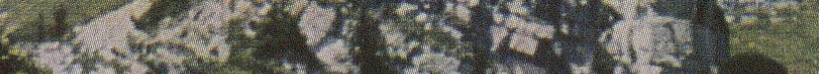

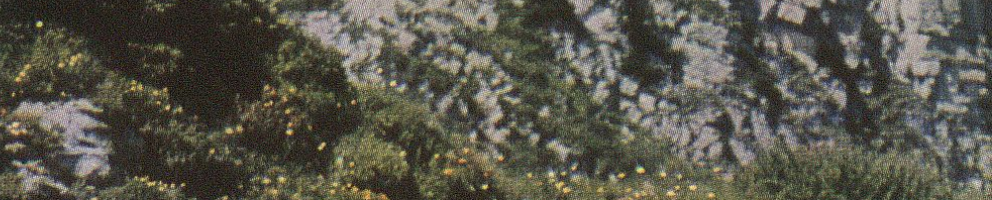
. 25.

4.

-5.

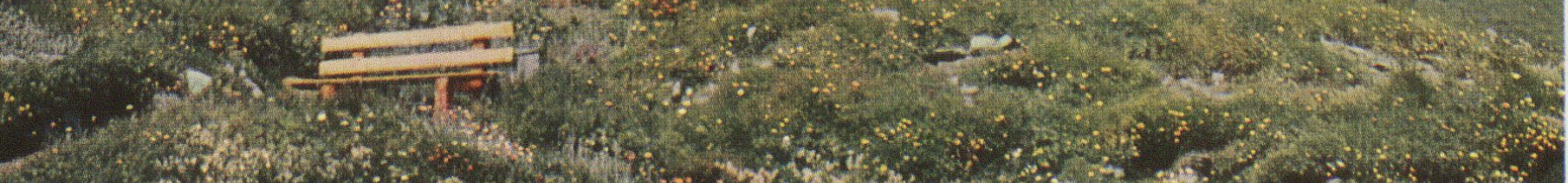

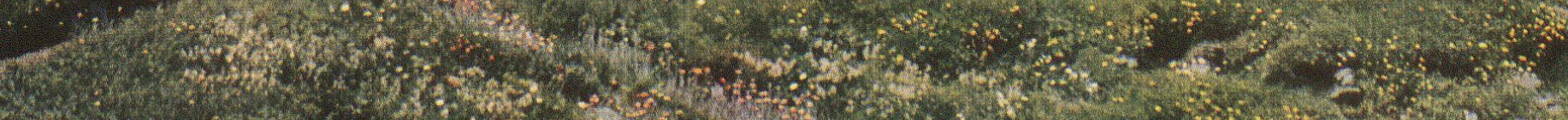

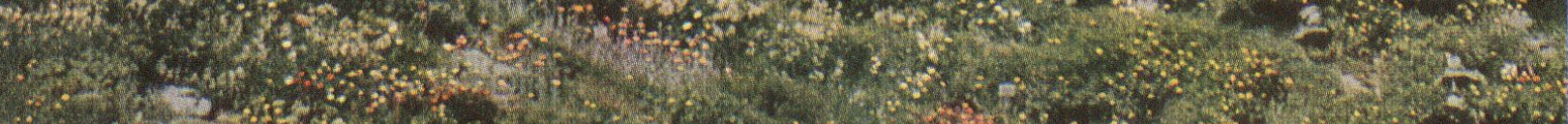

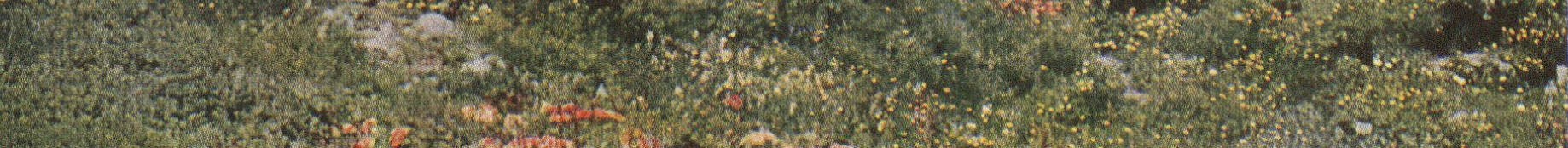

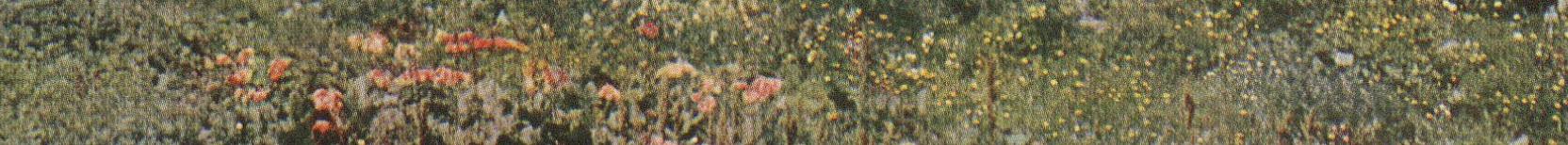

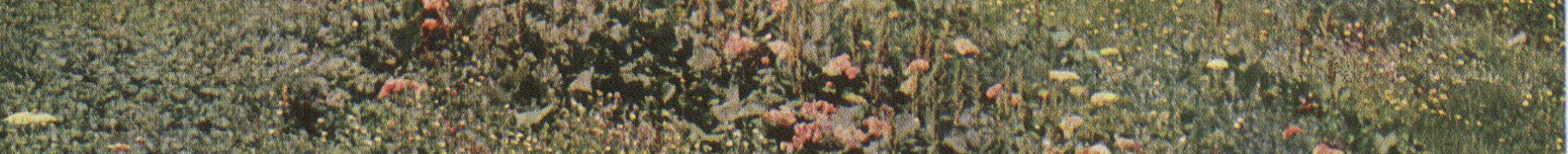

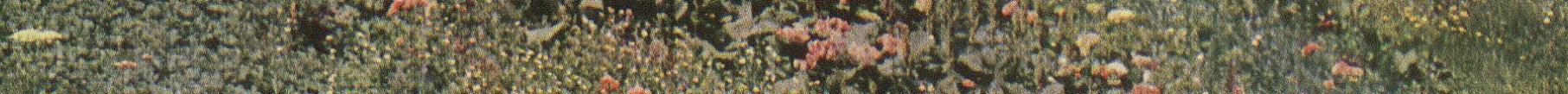

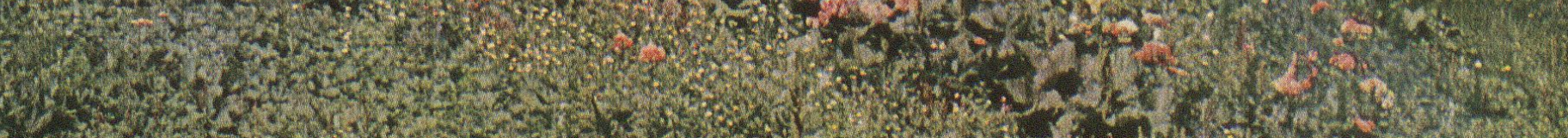

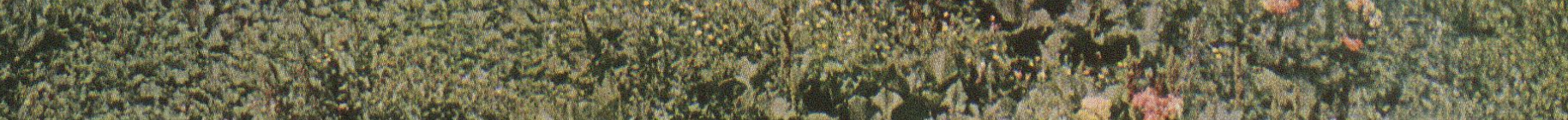

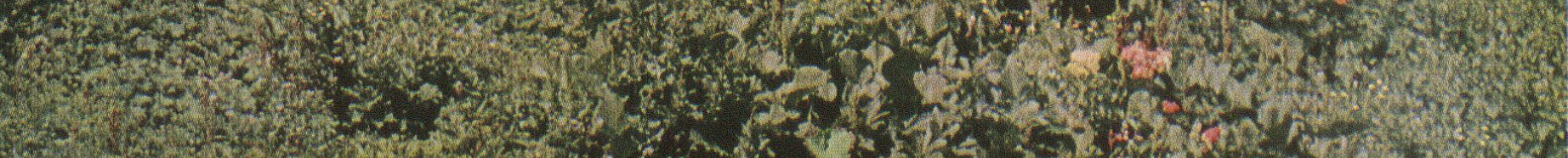

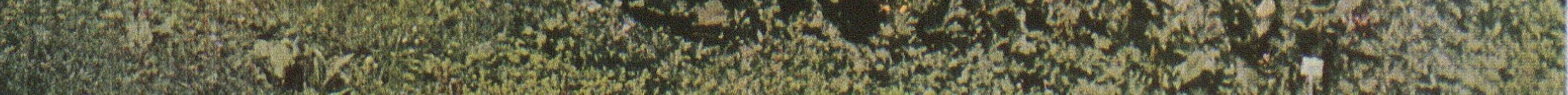

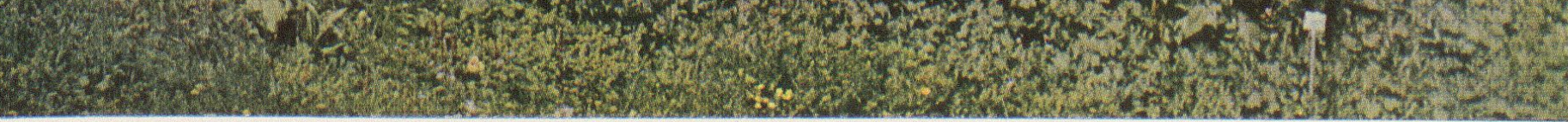




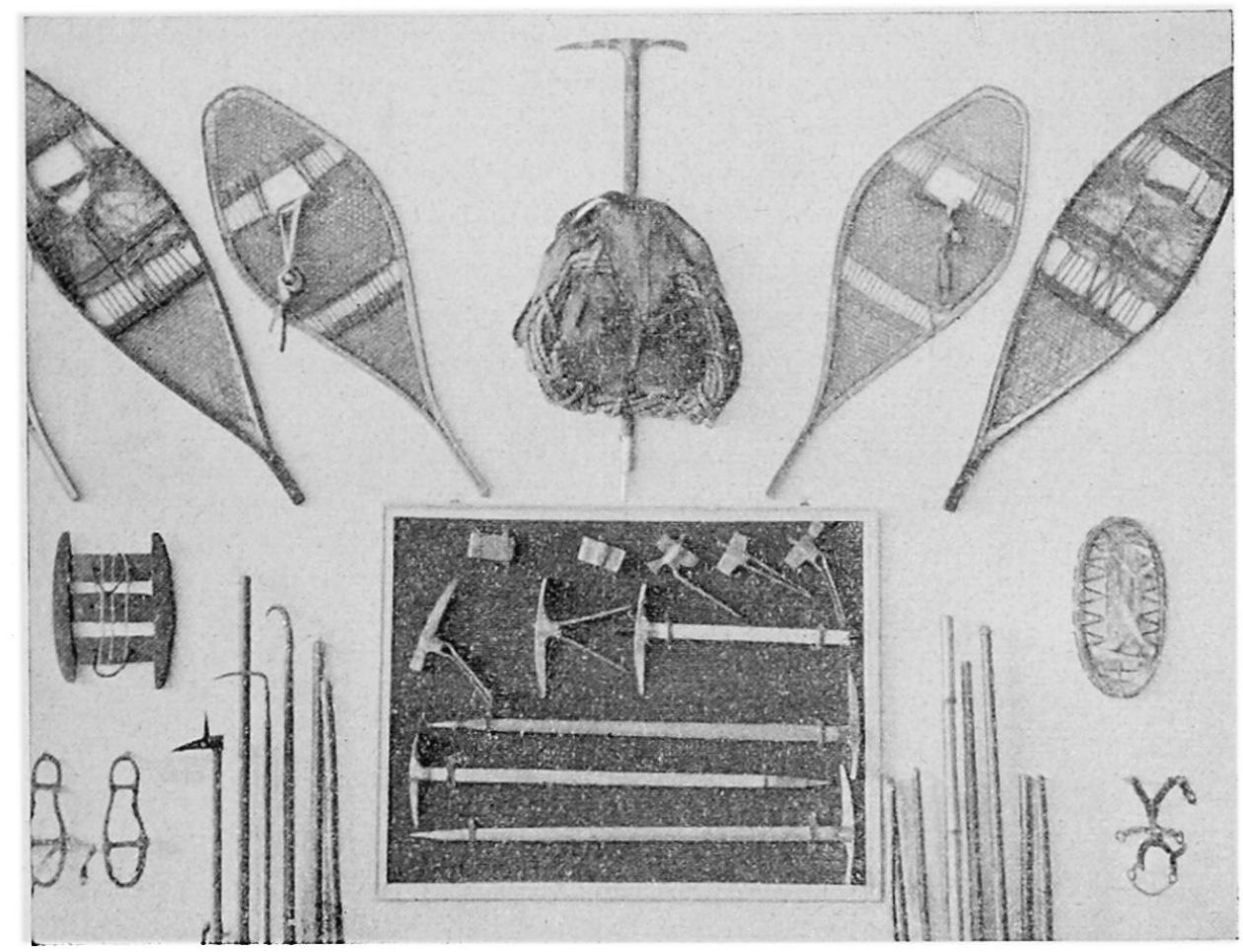

Abb. 2 Alpinistische Ausrüstung um 1900. Schneereifen, Bergstöcke, Werdegang eines Gletscherpickels, ältere Steigeisen. (Aus W. RYTZ: Fünfzig Jahre Schweizer. Alpines Museum, Bern 1955.) Beide Bilder mit freundlicher Erlaubnis der Direktion.

Das Museum verdient sicher einen Besuch; denn es will neben der Aufklärung über Werden und Vergehen der Alpen, ihre Schönheiten und Gefahren das Verständnis für die Besonderheiten der Natur, der Siedlung und der Lebensweise in den Alpen wecken.

LITERATUR: Rytz Walter, Prof. Dr.: 50 Jahre Schweizerisches Alpines Museum. Wissenschaftliche Mitteilungen des Schweiz. Alpinen Museums in Bern, Nr. 9, Verlag Schweiz. Alpines Museum 1955, 24 Seiten, 9 Aufnahmen, Fr. 1.-.

\section{CINQUANTENAIRE DU MUSÉE ALPIN SUISSE}

Le 9 juillet 1905, un musée alpin fut ouvert à Berne. Ses initiateurs étaient entre autres Paul Utinger et Rudolf Zeller, plus tard professeur de géographie à l'université de cette ville. Dès ses débuts, le musée se destina à devenir une exposition complète de tout ce qui concerne l'alpe. Cependant, au cours des ans, il réussit, grâce à l'aide de nombreux donateurs, à présenter aussi bien la nature (géologie, glaciologie, flore, faune) que la culture de la haute montagne ellemême (tourisme, habitations, trafic, folklore, économie, arts, etc.). Dans ce but, de nouveaux locaux au Kirchenfeld (depuis 1934) permirent d'agrandir et de compléter ce musée. Selon les principes de ses directeurs, particulièrement de l'actuel: Prof. Dr. W. RYTZ, ce musée se trouve en constante régénération. C'est pourquoi il offre toujours quelque chose de nouveau et peut être recommandé ici instamment aux visiteurs de Berne.

\section{JAHRE ALPENGARTEN SCHYNIGE PLATTE}

\section{HiLdegard LAMPRECHT}

Im Jahre 1930 konnte der Alpengarten auf der Schynigen Platte bei Interlaken zum ersten Mal seine Tore den Besuchern für den ganzen Sommer öffnen. Seither verzeichnete er jährlich eine stets wachsende Besucherzahl. Der Außenstehende wird sich kaum eine Vorstellung machen können von der vielen Arbeit, die Anlage und Unterhalt eines solchen botanischen Gartens erfordern. Es gab kein Lehrbuch über 